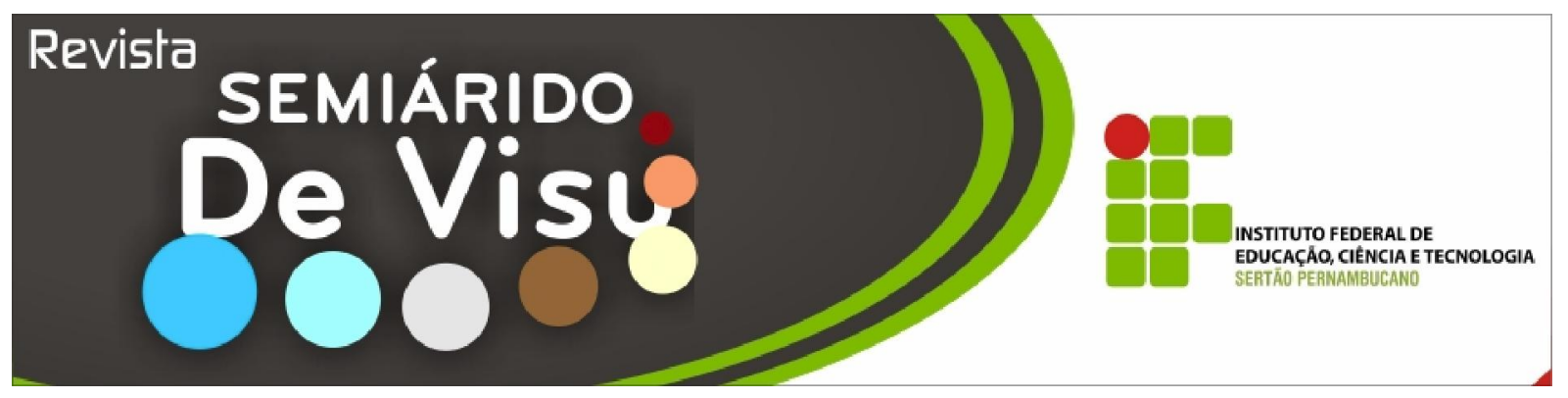

\title{
Ocorrência de Phakopsora euvitis Ono em folhas jovens de videira
}

\author{
Erbs Cintra de Souza Gomes ${ }^{1}$, Kedma Maria Silva Pinto ${ }^{1}$, Rodrigo Pereira Leite ${ }^{2}$, Fábio Júnior \\ Araújo Silva ${ }^{2}$, Janaína dos Reis Miranda ${ }^{1}$, Luciana Cordeiro do Nascimento ${ }^{1}$ \\ ${ }^{1}$ Programa de Pós-graduação em Agronomia, CCA/UFPB, Areia, PB. Rua Santa Rita, 130, apto. 03, Centro, Areia, PB. \\ CEP: 58397 000. E-mail: ectecnologo@hotmail.com \\ ${ }^{2}$ Laboratório de Fitopatologia, Centro de Ciências Agrárias - CCA/UFPB, Areia, PB
}

RESUMO: A ferrugem da videira, causada por Phakopsora euvitis Ono ocorre preferencialmente em folhas maduras, causando a desfolha precoce das plantas infectadas, comprometendo a maturação de frutos e ainda, de ramos para as próximas safras. Objetivou-se com este trabalho relatar a ocorrência de P. euvitis em folhas jovens de plantios comerciais de uva 'Isabel' no município de Natuba, PB. As amostras de folhas jovens foram coletadas em quatro diferentes áreas de produção e encaminhadas ao Laboratório de Fitopatologia do CCA/UFPB, para análise em microscopia óptica. A determinação do cálculo de incidência foi realizada de acordo com a metodologia de amostragem de doenças da videira definida pela Produção Integrada de Frutas - PIF de Uvas. Após a visualização em lâminas, confirmaram-se a presença de pústulas amareladas, características da ferrugem em amostras de folhas jovens de videira das quatro áreas avaliadas. A incidência média de ferrugem das quatro áreas avaliadas foi de 42,2\%.

Palavras-chave: Vitis labrusca, ferrugem, incidência.

\section{Ocurrence of in Phakopsora euvitis Ono young leaves of grapevine plants}

\begin{abstract}
Grapevine rust caused by Phakopsora euvitis Ono occurs preferentially in mature leaves, causing early defoliation of infected plants, affecting fruits and branches maturation in the following seasons. The objective of this study was to report the occurence of P. euvitis in young leaves of commercial planting of 'Isabel' grape plants. In Natuba, State of Paraíba, Brazil. Samples of young leaves were collected in four different production areas and sent to the Laboratory of Phytopathology, CCA/UFPB for optical microscopy analysis. The determination of the calculation of incidence was carried out according to sampling methodology of grapevine diseases defined by the Integrated Fruit Production: grape IFP. After viewing in blades, the presence of yellowing pustules was confirmed, characteristics of the rust in samples of young leaves of the vine of the four areas assessed. The incidence of rust rating of the four areas assessed was $42.2 \%$
\end{abstract}

Key Words: Vitis labrusca, rust, incidence 
Erbs Cintra de Souza Gomes et al.

cultura da videira (Vitis labrusca
L.) representa um dos pilares para
renda familiar dos pequenos produtores do município de Natuba, Paraíba, trazendo benefícios diretos aos produtores e ao município, que se destaca no cenário regional como único produtor de uvas do Estado (Souza et al., 2007).

Não obstante à realidade das principais regiões produtoras de uvas no mundo, a região de Natuba enfrenta sérios problemas fitossanitários que acometem diretamente a cultura, influenciando negativamente as características de produtividade e os aspectos de qualidade final dos produtos. Dentre as principais doenças relacionadas à cultura, destaca-se na região a ferrugem da videira (Phakopsora euvitis Ono) (Souza et al., 2007).

No Brasil, a ocorrência de P. euvitis foi constatada pela primeira vez em 2001, no município de Jandaia do Sul, PR, em um vinhedo comercial da variedade Itália (Tessmann et al., 2004). Posteriormente a doença foi relatada nos estados do Mato Grosso do Sul e São Paulo (Papa et al., 2003).

A doença ocorre geralmente em folhas maduras, causando a desfolha precoce das plantas infectadas (Tessmann et al., 2004) e pode prejudicar a maturação de frutos e, dos ramos para as próximas safras. As pústulas são formadas cinco a seis dias após a inoculação, em temperaturas de 16 a $30^{\circ} \mathrm{C}$, sendo as mínima, ótima e máxima para a germinação dos uredósporos são 8,24 e $32^{\circ} \mathrm{C}$, respectivamente e o período de incubação é mais longo, de 15 a 20 dias, em temperaturas inferiores a $16^{\circ} \mathrm{C}$ (Pearson \& Goheen,1988).

A ferrugem da videira é particularmente severa na pré-colheita em regiões de clima temperado, mas sua severidade é considerável durante todo o ano em regiões de clima subtropical. O ciclo de vida da espécie não é totalmente conhecido (Ono, 2000), embora mais rara no campo, em condições controladas o patógeno infecta também folhas novas (Tessman et al., 2007).

Neste sentido, objetivou-se com este trabalho relatar a ocorrência de $P$. euvitis, determinando os percentuais de infestação, em folhas jovens de videira 'Isabel' em condições de campo, no município de Natuba.

No primeiro semestre de 2009, amostras de uvas 'Isabel' ( . labrusca), constituídas de folhas jovens procedentes dos Brejos do Fervedouro e Jussaral, regiões produtoras do município de Natuba, apresentaram a sintomatologia característica da ferrugem da videira ( $P$. euvitis). Posteriormente, o material foi coletado e encaminhado ao Laboratório de Fitopatologia do Centro de Ciências Agrárias da Universidade Federal da Paraíba, para estudos morfológicos através de exame das estruturas do agente etiológico em microscopia óptica.

Em campo, realizou-se o monitoramento de quatro áreas de produção com intervalo máximo de 30 dias após a poda, para determinação da incidência média de $P$. euvitis em folhas jovens de videira. As avaliações seguiram as recomendações da Produção Integrada de Frutas - (PIF) (Brasil, 2008) os dados foram transformados em porcentagem de doença (\%) segundo o Índice de Doenças, definido por Campbell \& Madden (1990).

O exame do material coletado através da visualização em microscopia óptica revelou que as pústulas amareladas presentes nas folhas jovens de videira 'Isabel' (Figura 1), correspondem à sintomatologia de ferrugem da videira causada pelo agente etiológico $P$. euvitis (Ono, 2000). 
Erbs Cintra de Souza Gomes et al.

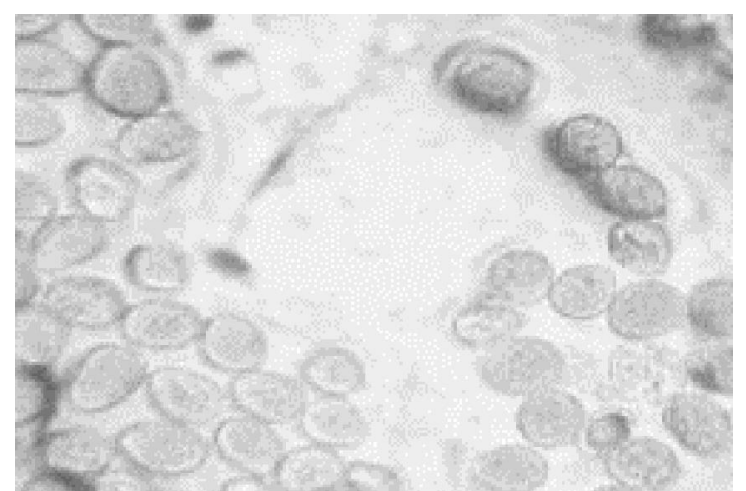

Figura 1. Uredósporos de Phakopsora euvitis em folhas de videira (Vitis labrusca L.)

A alta incidência de $P$. euvitis em folhas jovens nos parreirais da região caracteriza o aumento da severidade da doença ao longo dos ciclos. O amarelecimento e posterior seca das folhas infectadas resultam em desfolha precoce, exposição excessiva dos frutos ao sol e consequentemente, redução da qualidade comercial dos frutos. Das amostras, a maior incidência foi registrada nos pomares localizados no Brejo do Fervedouro - área 4 $(67,4 \%)$ (Figura 2). Os demais registros, áreas 1,2 e 3 foram observados em áreas comerciais do Brejo do Jussaral, região responsável pela maior produção do município.

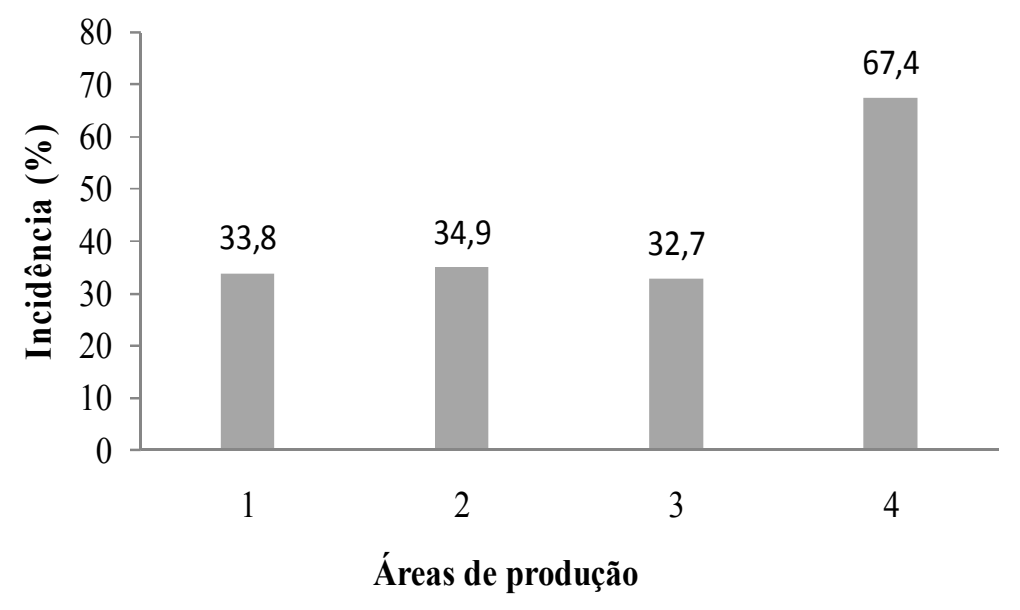

Figura 2. Incidência média de Phakopsora euvitis em folhas jovens de uvas 'Isabel' (Vitis labrusca) em quatro áreas de produção no município de Natuba, PB, 2009.

Contrariamente às afirmações de Tessman et al. (2007), observou-se em condições de campo a rápida disseminação do patógeno, o que pode ocasionado pelo inadequado manejo fitossanitário da doença adotado pelos produtores da região.

Assim, o presente trabalho comprovou a presença de $P$. euvitis em folhas jovens de videira 'Isabel' em condições de campo, comprometendo significativamente a produtividade das áreas e a sustentabilidade da produção de uvas no município de Natuba, PB.

\section{Referências}

BRASIL. Embrapa. Produção Integrada de Frutas. Normas técnicas específicas para a 
Erbs Cintra de Souza Gomes et al.

produção integrada de uvas finas de mesa. In: Manual de Monitoramento de doenças. Disponível em: http://www.cpatsa.embrapa.br/pif/uva/kit_Uva_ Doencas_edicao2.pdf Acesso em: 15 jun. 2008.

CAMPBELL, C.L. \& MADDEN, L.V. Introduction to plant disease epidemiology. New York. John Wiley \& Sons Inc. 1990.

ONO, Y. Taxonomy of the Phakopsora ampelopsidis species complex on vitaceous hosts in Ásia including a new species, P. euvitis. Mycologia, v.92, p.154-173, 2000.

PAPA, M. F. S.; CELOTO, M. Y. B; TOMQUELSKI, G. V; NARUZAWA, E. S. \& BOLIANI, A. C. Ocorrência de ferrugem da videira em São Paulo e Mato Grosso do Sul e controle químico em dois sistemas de condução. Fitopatologia Brasileira, v. 28, p. 320, 2003. Suplemento.

PEARSON, R.C. \& GOHEEN, A.C. Compendium of grapes disease. St. Paul: APS Press, 1988. 93p.

SOUZA, E.G.; OLIVEIRA, C.P. \& GOMES, F.S.L. Diagnóstico sócio-econômico das atividades de uva e banana de Natuba, Paraíba. BNB, 2007. 84p.

TESSMANN, D.J.; DIANESE, J.C.; GENTA, W.; VIDA, J.B. \& MAY-DE-MIO, L.L. Grape rust caused by Phakopsora euvitis, a new disease for Brazil. Fitopatologia Brasileira, v.29, n.3, p.338 2004.

TESSMANN, D.J.; VIDA, J.B.; GENTA, W. \& KISHINO, A.Y. Doenças e seu manejo. In: KISHINO, A.S.; CARVALHO, S.L.C.; ROBERTO, S.R. (Ed.). Viticultura Tropical: O sistema de produção do Paraná. Londrina: IAPAR, 2007. p.255-304. 